\title{
A Brief History of Kähler Geometry
}

\section{by Shing-Tung Yau*}

\section{Contents}

1 The Work of Riemann . . . . . . . . . . . . 1

2 Calabi Conjecture and Kähler-Einstein Metrics 4

2.1 Kähler-Einstein Metrics on Fano

Manifolds .............. 5

2.2 Balanced Metric and Strominger System 6

2.3 Questions of Kähler-Einstein Metrics in

Algebraic Geometry . . . . . . . . . . 7

2.3.1 Understanding of Kähler-Einstein

Metrics near Singularities . . . . 7

2.3.2 Kähler-Einstein Metrics on Quasiprojective Varieties and Sasakian-

Einstein Metrics . . . . . . . . 8

2.3.3 Compatification of Shimura Varieties ............ 8

2.3.4 Explicit Construction of KählerEinstein Metrics and Uniformization . . . . . . . . . . 10

2.3.5 Relation with Birational Geometry 11

3 Hermitian-Yang-Mills Connections . . . . . . . 12

3.1 Donaldson-Uhlenbeck-Yau Correspondence . . . . . . . . . . . . . . . . 12

3.2 Chern Number Inequalities and Characterization of Flat Bundles . . . . . . . . 13

3.3 Generalization to Non-Kähler and NonCompact Manifolds . . . . . . . . . . . 14

3.4 Analytic Criterions for Various Stability Conditions . . . . . . . . . . . . . . . 14

4 Mirror Symmetry . . . . . . . . . . . . . . . . 15

4.1 Counting of Curves . . . . . . . . . . 15

4.2 Mathematical Approaches to Mirror Symmetry . . . . . . . . . . . . . . . 15

5 Future Directions in Mathematical Physics and Arithmetic Geometry . . . . . . . . 16

References . . . . . . . . . . . . . . . . . 17

* Department of Mathematics, Harvard University, Cambridge, Massachusetts, U.S.A.

E-mail: yau@math.harvard.edu

\section{The Work of Riemann}

Riemann was one of the founders of complex analysis, along with Cauchy. Riemann pioneered several directions in the subject of holomorphic functions:

1. The idea of using differential equations and variational principle. The major work here is the Cauchy-Riemann equation, and the creation of Dirichlet principle to solve the boundary value problem for harmonic functions. (It took several great mathematicians, such as David Hilbert, to complete this work of Riemann.)

2. He gave the proof of the Riemann mapping theorem for simply connected domains. This theory of uniformization theorems has been extremely influential. There are methods based on various approaches, including methods of partial differential equations, hypergeometric functions and algebraic geometry. A natural generalization is to understand the moduli space of Riemann surfaces where Riemann made an important contribution by showing that it is a complex variety with dimension $3 g-3$.

3. The idea of using geometry to understand multivalued holomorphic functions, where he looked at the largest domain that a multivalued holomorphic function can define. He created the concept of Riemann surfaces, where he studied their topology and their moduli space. In fact, he introduced the concept of connectivity of space by cutting Riemann surface into pieces. The concept of Betti number was introduced by him for spaces in arbitrary dimension.

The idea of understanding analytic problems through topology or geometry has far-reaching consequences. It influenced the later works of Poincaré, Picard, Lefstchetz, Hodge and others. 


\section{A Brief History of Kähler Geometry}

\section{by Shing-Tung Yau*}

\section{Contents}

1 The Work of Riemann . . . . . . . . . . . . 1

2 Calabi Conjecture and Kähler-Einstein Metrics 4

2.1 Kähler-Einstein Metrics on Fano

Manifolds .............. 5

2.2 Balanced Metric and Strominger System 6

2.3 Questions of Kähler-Einstein Metrics in

Algebraic Geometry . . . . . . . . . . 7

2.3.1 Understanding of Kähler-Einstein

Metrics near Singularities . . . . 7

2.3.2 Kähler-Einstein Metrics on Quasiprojective Varieties and Sasakian-

Einstein Metrics . . . . . . . . 8

2.3.3 Compatification of Shimura Varieties ............ 8

2.3.4 Explicit Construction of KählerEinstein Metrics and Uniformization . . . . . . . . . . 10

2.3.5 Relation with Birational Geometry 11

3 Hermitian-Yang-Mills Connections . . . . . . . 12

3.1 Donaldson-Uhlenbeck-Yau Correspondence . . . . . . . . . . . . . . . . 12

3.2 Chern Number Inequalities and Characterization of Flat Bundles . . . . . . . . 13

3.3 Generalization to Non-Kähler and NonCompact Manifolds . . . . . . . . . . . 14

3.4 Analytic Criterions for Various Stability Conditions . . . . . . . . . . . . . . . 14

4 Mirror Symmetry . . . . . . . . . . . . . . . . 15

4.1 Counting of Curves . . . . . . . . . . 15

4.2 Mathematical Approaches to Mirror Symmetry . . . . . . . . . . . . . . . 15

5 Future Directions in Mathematical Physics and Arithmetic Geometry . . . . . . . . 16

References . . . . . . . . . . . . . . . . . 17

* Department of Mathematics, Harvard University, Cambridge, Massachusetts, U.S.A.

E-mail: yau@math.harvard.edu

\section{The Work of Riemann}

Riemann was one of the founders of complex analysis, along with Cauchy. Riemann pioneered several directions in the subject of holomorphic functions:

1. The idea of using differential equations and variational principle. The major work here is the Cauchy-Riemann equation, and the creation of Dirichlet principle to solve the boundary value problem for harmonic functions. (It took several great mathematicians, such as David Hilbert, to complete this work of Riemann.)

2. He gave the proof of the Riemann mapping theorem for simply connected domains. This theory of uniformization theorems has been extremely influential. There are methods based on various approaches, including methods of partial differential equations, hypergeometric functions and algebraic geometry. A natural generalization is to understand the moduli space of Riemann surfaces where Riemann made an important contribution by showing that it is a complex variety with dimension $3 g-3$.

3. The idea of using geometry to understand multivalued holomorphic functions, where he looked at the largest domain that a multivalued holomorphic function can define. He created the concept of Riemann surfaces, where he studied their topology and their moduli space. In fact, he introduced the concept of connectivity of space by cutting Riemann surface into pieces. The concept of Betti number was introduced by him for spaces in arbitrary dimension.

The idea of understanding analytic problems through topology or geometry has far-reaching consequences. It influenced the later works of Poincaré, Picard, Lefstchetz, Hodge and others. 


\section{A Brief History of Kähler Geometry}

\section{by Shing-Tung Yau*}

\section{Contents}

1 The Work of Riemann . . . . . . . . . . . . 1

2 Calabi Conjecture and Kähler-Einstein Metrics 4

2.1 Kähler-Einstein Metrics on Fano

Manifolds .............. 5

2.2 Balanced Metric and Strominger System 6

2.3 Questions of Kähler-Einstein Metrics in

Algebraic Geometry . . . . . . . . . . 7

2.3.1 Understanding of Kähler-Einstein

Metrics near Singularities . . . . 7

2.3.2 Kähler-Einstein Metrics on Quasiprojective Varieties and Sasakian-

Einstein Metrics . . . . . . . . 8

2.3.3 Compatification of Shimura Varieties ............ 8

2.3.4 Explicit Construction of KählerEinstein Metrics and Uniformization . . . . . . . . . . 10

2.3.5 Relation with Birational Geometry 11

3 Hermitian-Yang-Mills Connections . . . . . . . 12

3.1 Donaldson-Uhlenbeck-Yau Correspondence . . . . . . . . . . . . . . . . 12

3.2 Chern Number Inequalities and Characterization of Flat Bundles . . . . . . . . 13

3.3 Generalization to Non-Kähler and NonCompact Manifolds . . . . . . . . . . . 14

3.4 Analytic Criterions for Various Stability Conditions . . . . . . . . . . . . . . . 14

4 Mirror Symmetry . . . . . . . . . . . . . . . . 15

4.1 Counting of Curves . . . . . . . . . . 15

4.2 Mathematical Approaches to Mirror Symmetry . . . . . . . . . . . . . . . 15

5 Future Directions in Mathematical Physics and Arithmetic Geometry . . . . . . . . 16

References . . . . . . . . . . . . . . . . . 17

* Department of Mathematics, Harvard University, Cambridge, Massachusetts, U.S.A.

E-mail: yau@math.harvard.edu

\section{The Work of Riemann}

Riemann was one of the founders of complex analysis, along with Cauchy. Riemann pioneered several directions in the subject of holomorphic functions:

1. The idea of using differential equations and variational principle. The major work here is the Cauchy-Riemann equation, and the creation of Dirichlet principle to solve the boundary value problem for harmonic functions. (It took several great mathematicians, such as David Hilbert, to complete this work of Riemann.)

2. He gave the proof of the Riemann mapping theorem for simply connected domains. This theory of uniformization theorems has been extremely influential. There are methods based on various approaches, including methods of partial differential equations, hypergeometric functions and algebraic geometry. A natural generalization is to understand the moduli space of Riemann surfaces where Riemann made an important contribution by showing that it is a complex variety with dimension $3 g-3$.

3. The idea of using geometry to understand multivalued holomorphic functions, where he looked at the largest domain that a multivalued holomorphic function can define. He created the concept of Riemann surfaces, where he studied their topology and their moduli space. In fact, he introduced the concept of connectivity of space by cutting Riemann surface into pieces. The concept of Betti number was introduced by him for spaces in arbitrary dimension.

The idea of understanding analytic problems through topology or geometry has far-reaching consequences. It influenced the later works of Poincaré, Picard, Lefstchetz, Hodge and others. 


\section{A Brief History of Kähler Geometry}

\section{by Shing-Tung Yau*}

\section{Contents}

1 The Work of Riemann . . . . . . . . . . . . 1

2 Calabi Conjecture and Kähler-Einstein Metrics 4

2.1 Kähler-Einstein Metrics on Fano

Manifolds .............. 5

2.2 Balanced Metric and Strominger System 6

2.3 Questions of Kähler-Einstein Metrics in

Algebraic Geometry . . . . . . . . . . 7

2.3.1 Understanding of Kähler-Einstein

Metrics near Singularities . . . . 7

2.3.2 Kähler-Einstein Metrics on Quasiprojective Varieties and Sasakian-

Einstein Metrics . . . . . . . . 8

2.3.3 Compatification of Shimura Varieties ............ 8

2.3.4 Explicit Construction of KählerEinstein Metrics and Uniformization . . . . . . . . . . 10

2.3.5 Relation with Birational Geometry 11

3 Hermitian-Yang-Mills Connections . . . . . . . 12

3.1 Donaldson-Uhlenbeck-Yau Correspondence . . . . . . . . . . . . . . . . 12

3.2 Chern Number Inequalities and Characterization of Flat Bundles . . . . . . . . 13

3.3 Generalization to Non-Kähler and NonCompact Manifolds . . . . . . . . . . . 14

3.4 Analytic Criterions for Various Stability Conditions . . . . . . . . . . . . . . . 14

4 Mirror Symmetry . . . . . . . . . . . . . . . . 15

4.1 Counting of Curves . . . . . . . . . . 15

4.2 Mathematical Approaches to Mirror Symmetry . . . . . . . . . . . . . . . 15

5 Future Directions in Mathematical Physics and Arithmetic Geometry . . . . . . . . 16

References . . . . . . . . . . . . . . . . . 17

* Department of Mathematics, Harvard University, Cambridge, Massachusetts, U.S.A.

E-mail: yau@math.harvard.edu

\section{The Work of Riemann}

Riemann was one of the founders of complex analysis, along with Cauchy. Riemann pioneered several directions in the subject of holomorphic functions:

1. The idea of using differential equations and variational principle. The major work here is the Cauchy-Riemann equation, and the creation of Dirichlet principle to solve the boundary value problem for harmonic functions. (It took several great mathematicians, such as David Hilbert, to complete this work of Riemann.)

2. He gave the proof of the Riemann mapping theorem for simply connected domains. This theory of uniformization theorems has been extremely influential. There are methods based on various approaches, including methods of partial differential equations, hypergeometric functions and algebraic geometry. A natural generalization is to understand the moduli space of Riemann surfaces where Riemann made an important contribution by showing that it is a complex variety with dimension $3 g-3$.

3. The idea of using geometry to understand multivalued holomorphic functions, where he looked at the largest domain that a multivalued holomorphic function can define. He created the concept of Riemann surfaces, where he studied their topology and their moduli space. In fact, he introduced the concept of connectivity of space by cutting Riemann surface into pieces. The concept of Betti number was introduced by him for spaces in arbitrary dimension.

The idea of understanding analytic problems through topology or geometry has far-reaching consequences. It influenced the later works of Poincaré, Picard, Lefstchetz, Hodge and others. 


\section{A Brief History of Kähler Geometry}

\section{by Shing-Tung Yau*}

\section{Contents}

1 The Work of Riemann . . . . . . . . . . . . 1

2 Calabi Conjecture and Kähler-Einstein Metrics 4

2.1 Kähler-Einstein Metrics on Fano

Manifolds .............. 5

2.2 Balanced Metric and Strominger System 6

2.3 Questions of Kähler-Einstein Metrics in

Algebraic Geometry . . . . . . . . . . 7

2.3.1 Understanding of Kähler-Einstein

Metrics near Singularities . . . . 7

2.3.2 Kähler-Einstein Metrics on Quasiprojective Varieties and Sasakian-

Einstein Metrics . . . . . . . . 8

2.3.3 Compatification of Shimura Varieties ............ 8

2.3.4 Explicit Construction of KählerEinstein Metrics and Uniformization . . . . . . . . . . 10

2.3.5 Relation with Birational Geometry 11

3 Hermitian-Yang-Mills Connections . . . . . . . 12

3.1 Donaldson-Uhlenbeck-Yau Correspondence . . . . . . . . . . . . . . . . 12

3.2 Chern Number Inequalities and Characterization of Flat Bundles . . . . . . . . 13

3.3 Generalization to Non-Kähler and NonCompact Manifolds . . . . . . . . . . . 14

3.4 Analytic Criterions for Various Stability Conditions . . . . . . . . . . . . . . . 14

4 Mirror Symmetry . . . . . . . . . . . . . . . . 15

4.1 Counting of Curves . . . . . . . . . . 15

4.2 Mathematical Approaches to Mirror Symmetry . . . . . . . . . . . . . . . 15

5 Future Directions in Mathematical Physics and Arithmetic Geometry . . . . . . . . 16

References . . . . . . . . . . . . . . . . . 17

* Department of Mathematics, Harvard University, Cambridge, Massachusetts, U.S.A.

E-mail: yau@math.harvard.edu

\section{The Work of Riemann}

Riemann was one of the founders of complex analysis, along with Cauchy. Riemann pioneered several directions in the subject of holomorphic functions:

1. The idea of using differential equations and variational principle. The major work here is the Cauchy-Riemann equation, and the creation of Dirichlet principle to solve the boundary value problem for harmonic functions. (It took several great mathematicians, such as David Hilbert, to complete this work of Riemann.)

2. He gave the proof of the Riemann mapping theorem for simply connected domains. This theory of uniformization theorems has been extremely influential. There are methods based on various approaches, including methods of partial differential equations, hypergeometric functions and algebraic geometry. A natural generalization is to understand the moduli space of Riemann surfaces where Riemann made an important contribution by showing that it is a complex variety with dimension $3 g-3$.

3. The idea of using geometry to understand multivalued holomorphic functions, where he looked at the largest domain that a multivalued holomorphic function can define. He created the concept of Riemann surfaces, where he studied their topology and their moduli space. In fact, he introduced the concept of connectivity of space by cutting Riemann surface into pieces. The concept of Betti number was introduced by him for spaces in arbitrary dimension.

The idea of understanding analytic problems through topology or geometry has far-reaching consequences. It influenced the later works of Poincaré, Picard, Lefstchetz, Hodge and others. 


\section{A Brief History of Kähler Geometry}

\section{by Shing-Tung Yau*}

\section{Contents}

1 The Work of Riemann . . . . . . . . . . . . 1

2 Calabi Conjecture and Kähler-Einstein Metrics 4

2.1 Kähler-Einstein Metrics on Fano

Manifolds .............. 5

2.2 Balanced Metric and Strominger System 6

2.3 Questions of Kähler-Einstein Metrics in

Algebraic Geometry . . . . . . . . . . 7

2.3.1 Understanding of Kähler-Einstein

Metrics near Singularities . . . . 7

2.3.2 Kähler-Einstein Metrics on Quasiprojective Varieties and Sasakian-

Einstein Metrics . . . . . . . . 8

2.3.3 Compatification of Shimura Varieties ............ 8

2.3.4 Explicit Construction of KählerEinstein Metrics and Uniformization . . . . . . . . . . 10

2.3.5 Relation with Birational Geometry 11

3 Hermitian-Yang-Mills Connections . . . . . . . 12

3.1 Donaldson-Uhlenbeck-Yau Correspondence . . . . . . . . . . . . . . . . 12

3.2 Chern Number Inequalities and Characterization of Flat Bundles . . . . . . . . 13

3.3 Generalization to Non-Kähler and NonCompact Manifolds . . . . . . . . . . . 14

3.4 Analytic Criterions for Various Stability Conditions . . . . . . . . . . . . . . . 14

4 Mirror Symmetry . . . . . . . . . . . . . . . . 15

4.1 Counting of Curves . . . . . . . . . . 15

4.2 Mathematical Approaches to Mirror Symmetry . . . . . . . . . . . . . . . 15

5 Future Directions in Mathematical Physics and Arithmetic Geometry . . . . . . . . 16

References . . . . . . . . . . . . . . . . . 17

* Department of Mathematics, Harvard University, Cambridge, Massachusetts, U.S.A.

E-mail: yau@math.harvard.edu

\section{The Work of Riemann}

Riemann was one of the founders of complex analysis, along with Cauchy. Riemann pioneered several directions in the subject of holomorphic functions:

1. The idea of using differential equations and variational principle. The major work here is the Cauchy-Riemann equation, and the creation of Dirichlet principle to solve the boundary value problem for harmonic functions. (It took several great mathematicians, such as David Hilbert, to complete this work of Riemann.)

2. He gave the proof of the Riemann mapping theorem for simply connected domains. This theory of uniformization theorems has been extremely influential. There are methods based on various approaches, including methods of partial differential equations, hypergeometric functions and algebraic geometry. A natural generalization is to understand the moduli space of Riemann surfaces where Riemann made an important contribution by showing that it is a complex variety with dimension $3 g-3$.

3. The idea of using geometry to understand multivalued holomorphic functions, where he looked at the largest domain that a multivalued holomorphic function can define. He created the concept of Riemann surfaces, where he studied their topology and their moduli space. In fact, he introduced the concept of connectivity of space by cutting Riemann surface into pieces. The concept of Betti number was introduced by him for spaces in arbitrary dimension.

The idea of understanding analytic problems through topology or geometry has far-reaching consequences. It influenced the later works of Poincaré, Picard, Lefstchetz, Hodge and others. 


\section{A Brief History of Kähler Geometry}

\section{by Shing-Tung Yau*}

\section{Contents}

1 The Work of Riemann . . . . . . . . . . . . 1

2 Calabi Conjecture and Kähler-Einstein Metrics 4

2.1 Kähler-Einstein Metrics on Fano

Manifolds .............. 5

2.2 Balanced Metric and Strominger System 6

2.3 Questions of Kähler-Einstein Metrics in

Algebraic Geometry . . . . . . . . . . 7

2.3.1 Understanding of Kähler-Einstein

Metrics near Singularities . . . . 7

2.3.2 Kähler-Einstein Metrics on Quasiprojective Varieties and Sasakian-

Einstein Metrics . . . . . . . . 8

2.3.3 Compatification of Shimura Varieties ............ 8

2.3.4 Explicit Construction of KählerEinstein Metrics and Uniformization . . . . . . . . . . 10

2.3.5 Relation with Birational Geometry 11

3 Hermitian-Yang-Mills Connections . . . . . . . 12

3.1 Donaldson-Uhlenbeck-Yau Correspondence . . . . . . . . . . . . . . . . 12

3.2 Chern Number Inequalities and Characterization of Flat Bundles . . . . . . . . 13

3.3 Generalization to Non-Kähler and NonCompact Manifolds . . . . . . . . . . . 14

3.4 Analytic Criterions for Various Stability Conditions . . . . . . . . . . . . . . . 14

4 Mirror Symmetry . . . . . . . . . . . . . . . . 15

4.1 Counting of Curves . . . . . . . . . . 15

4.2 Mathematical Approaches to Mirror Symmetry . . . . . . . . . . . . . . . 15

5 Future Directions in Mathematical Physics and Arithmetic Geometry . . . . . . . . 16

References . . . . . . . . . . . . . . . . . 17

* Department of Mathematics, Harvard University, Cambridge, Massachusetts, U.S.A.

E-mail: yau@math.harvard.edu

\section{The Work of Riemann}

Riemann was one of the founders of complex analysis, along with Cauchy. Riemann pioneered several directions in the subject of holomorphic functions:

1. The idea of using differential equations and variational principle. The major work here is the Cauchy-Riemann equation, and the creation of Dirichlet principle to solve the boundary value problem for harmonic functions. (It took several great mathematicians, such as David Hilbert, to complete this work of Riemann.)

2. He gave the proof of the Riemann mapping theorem for simply connected domains. This theory of uniformization theorems has been extremely influential. There are methods based on various approaches, including methods of partial differential equations, hypergeometric functions and algebraic geometry. A natural generalization is to understand the moduli space of Riemann surfaces where Riemann made an important contribution by showing that it is a complex variety with dimension $3 g-3$.

3. The idea of using geometry to understand multivalued holomorphic functions, where he looked at the largest domain that a multivalued holomorphic function can define. He created the concept of Riemann surfaces, where he studied their topology and their moduli space. In fact, he introduced the concept of connectivity of space by cutting Riemann surface into pieces. The concept of Betti number was introduced by him for spaces in arbitrary dimension.

The idea of understanding analytic problems through topology or geometry has far-reaching consequences. It influenced the later works of Poincaré, Picard, Lefstchetz, Hodge and others. 


\section{A Brief History of Kähler Geometry}

\section{by Shing-Tung Yau*}

\section{Contents}

1 The Work of Riemann . . . . . . . . . . . . 1

2 Calabi Conjecture and Kähler-Einstein Metrics 4

2.1 Kähler-Einstein Metrics on Fano

Manifolds .............. 5

2.2 Balanced Metric and Strominger System 6

2.3 Questions of Kähler-Einstein Metrics in

Algebraic Geometry . . . . . . . . . . 7

2.3.1 Understanding of Kähler-Einstein

Metrics near Singularities . . . . 7

2.3.2 Kähler-Einstein Metrics on Quasiprojective Varieties and Sasakian-

Einstein Metrics . . . . . . . . 8

2.3.3 Compatification of Shimura Varieties ............ 8

2.3.4 Explicit Construction of KählerEinstein Metrics and Uniformization . . . . . . . . . . 10

2.3.5 Relation with Birational Geometry 11

3 Hermitian-Yang-Mills Connections . . . . . . . 12

3.1 Donaldson-Uhlenbeck-Yau Correspondence . . . . . . . . . . . . . . . . 12

3.2 Chern Number Inequalities and Characterization of Flat Bundles . . . . . . . . 13

3.3 Generalization to Non-Kähler and NonCompact Manifolds . . . . . . . . . . . 14

3.4 Analytic Criterions for Various Stability Conditions . . . . . . . . . . . . . . . 14

4 Mirror Symmetry . . . . . . . . . . . . . . . . 15

4.1 Counting of Curves . . . . . . . . . . 15

4.2 Mathematical Approaches to Mirror Symmetry . . . . . . . . . . . . . . . 15

5 Future Directions in Mathematical Physics and Arithmetic Geometry . . . . . . . . 16

References . . . . . . . . . . . . . . . . . 17

* Department of Mathematics, Harvard University, Cambridge, Massachusetts, U.S.A.

E-mail: yau@math.harvard.edu

\section{The Work of Riemann}

Riemann was one of the founders of complex analysis, along with Cauchy. Riemann pioneered several directions in the subject of holomorphic functions:

1. The idea of using differential equations and variational principle. The major work here is the Cauchy-Riemann equation, and the creation of Dirichlet principle to solve the boundary value problem for harmonic functions. (It took several great mathematicians, such as David Hilbert, to complete this work of Riemann.)

2. He gave the proof of the Riemann mapping theorem for simply connected domains. This theory of uniformization theorems has been extremely influential. There are methods based on various approaches, including methods of partial differential equations, hypergeometric functions and algebraic geometry. A natural generalization is to understand the moduli space of Riemann surfaces where Riemann made an important contribution by showing that it is a complex variety with dimension $3 g-3$.

3. The idea of using geometry to understand multivalued holomorphic functions, where he looked at the largest domain that a multivalued holomorphic function can define. He created the concept of Riemann surfaces, where he studied their topology and their moduli space. In fact, he introduced the concept of connectivity of space by cutting Riemann surface into pieces. The concept of Betti number was introduced by him for spaces in arbitrary dimension.

The idea of understanding analytic problems through topology or geometry has far-reaching consequences. It influenced the later works of Poincaré, Picard, Lefstchetz, Hodge and others. 


\section{A Brief History of Kähler Geometry}

\section{by Shing-Tung Yau*}

\section{Contents}

1 The Work of Riemann . . . . . . . . . . . . 1

2 Calabi Conjecture and Kähler-Einstein Metrics 4

2.1 Kähler-Einstein Metrics on Fano

Manifolds .............. 5

2.2 Balanced Metric and Strominger System 6

2.3 Questions of Kähler-Einstein Metrics in

Algebraic Geometry . . . . . . . . . . 7

2.3.1 Understanding of Kähler-Einstein

Metrics near Singularities . . . . 7

2.3.2 Kähler-Einstein Metrics on Quasiprojective Varieties and Sasakian-

Einstein Metrics . . . . . . . . 8

2.3.3 Compatification of Shimura Varieties ............ 8

2.3.4 Explicit Construction of KählerEinstein Metrics and Uniformization . . . . . . . . . . 10

2.3.5 Relation with Birational Geometry 11

3 Hermitian-Yang-Mills Connections . . . . . . . 12

3.1 Donaldson-Uhlenbeck-Yau Correspondence . . . . . . . . . . . . . . . . 12

3.2 Chern Number Inequalities and Characterization of Flat Bundles . . . . . . . . 13

3.3 Generalization to Non-Kähler and NonCompact Manifolds . . . . . . . . . . . 14

3.4 Analytic Criterions for Various Stability Conditions . . . . . . . . . . . . . . . 14

4 Mirror Symmetry . . . . . . . . . . . . . . . . 15

4.1 Counting of Curves . . . . . . . . . . 15

4.2 Mathematical Approaches to Mirror Symmetry . . . . . . . . . . . . . . . 15

5 Future Directions in Mathematical Physics and Arithmetic Geometry . . . . . . . . 16

References . . . . . . . . . . . . . . . . . 17

* Department of Mathematics, Harvard University, Cambridge, Massachusetts, U.S.A.

E-mail: yau@math.harvard.edu

\section{The Work of Riemann}

Riemann was one of the founders of complex analysis, along with Cauchy. Riemann pioneered several directions in the subject of holomorphic functions:

1. The idea of using differential equations and variational principle. The major work here is the Cauchy-Riemann equation, and the creation of Dirichlet principle to solve the boundary value problem for harmonic functions. (It took several great mathematicians, such as David Hilbert, to complete this work of Riemann.)

2. He gave the proof of the Riemann mapping theorem for simply connected domains. This theory of uniformization theorems has been extremely influential. There are methods based on various approaches, including methods of partial differential equations, hypergeometric functions and algebraic geometry. A natural generalization is to understand the moduli space of Riemann surfaces where Riemann made an important contribution by showing that it is a complex variety with dimension $3 g-3$.

3. The idea of using geometry to understand multivalued holomorphic functions, where he looked at the largest domain that a multivalued holomorphic function can define. He created the concept of Riemann surfaces, where he studied their topology and their moduli space. In fact, he introduced the concept of connectivity of space by cutting Riemann surface into pieces. The concept of Betti number was introduced by him for spaces in arbitrary dimension.

The idea of understanding analytic problems through topology or geometry has far-reaching consequences. It influenced the later works of Poincaré, Picard, Lefstchetz, Hodge and others. 


\section{A Brief History of Kähler Geometry}

\section{by Shing-Tung Yau*}

\section{Contents}

1 The Work of Riemann . . . . . . . . . . . . 1

2 Calabi Conjecture and Kähler-Einstein Metrics 4

2.1 Kähler-Einstein Metrics on Fano

Manifolds .............. 5

2.2 Balanced Metric and Strominger System 6

2.3 Questions of Kähler-Einstein Metrics in

Algebraic Geometry . . . . . . . . . . 7

2.3.1 Understanding of Kähler-Einstein

Metrics near Singularities . . . . 7

2.3.2 Kähler-Einstein Metrics on Quasiprojective Varieties and Sasakian-

Einstein Metrics . . . . . . . . 8

2.3.3 Compatification of Shimura Varieties ............ 8

2.3.4 Explicit Construction of KählerEinstein Metrics and Uniformization . . . . . . . . . . 10

2.3.5 Relation with Birational Geometry 11

3 Hermitian-Yang-Mills Connections . . . . . . . 12

3.1 Donaldson-Uhlenbeck-Yau Correspondence . . . . . . . . . . . . . . . . 12

3.2 Chern Number Inequalities and Characterization of Flat Bundles . . . . . . . . 13

3.3 Generalization to Non-Kähler and NonCompact Manifolds . . . . . . . . . . . 14

3.4 Analytic Criterions for Various Stability Conditions . . . . . . . . . . . . . . . 14

4 Mirror Symmetry . . . . . . . . . . . . . . . . 15

4.1 Counting of Curves . . . . . . . . . . 15

4.2 Mathematical Approaches to Mirror Symmetry . . . . . . . . . . . . . . . 15

5 Future Directions in Mathematical Physics and Arithmetic Geometry . . . . . . . . 16

References . . . . . . . . . . . . . . . . . 17

* Department of Mathematics, Harvard University, Cambridge, Massachusetts, U.S.A.

E-mail: yau@math.harvard.edu

\section{The Work of Riemann}

Riemann was one of the founders of complex analysis, along with Cauchy. Riemann pioneered several directions in the subject of holomorphic functions:

1. The idea of using differential equations and variational principle. The major work here is the Cauchy-Riemann equation, and the creation of Dirichlet principle to solve the boundary value problem for harmonic functions. (It took several great mathematicians, such as David Hilbert, to complete this work of Riemann.)

2. He gave the proof of the Riemann mapping theorem for simply connected domains. This theory of uniformization theorems has been extremely influential. There are methods based on various approaches, including methods of partial differential equations, hypergeometric functions and algebraic geometry. A natural generalization is to understand the moduli space of Riemann surfaces where Riemann made an important contribution by showing that it is a complex variety with dimension $3 g-3$.

3. The idea of using geometry to understand multivalued holomorphic functions, where he looked at the largest domain that a multivalued holomorphic function can define. He created the concept of Riemann surfaces, where he studied their topology and their moduli space. In fact, he introduced the concept of connectivity of space by cutting Riemann surface into pieces. The concept of Betti number was introduced by him for spaces in arbitrary dimension.

The idea of understanding analytic problems through topology or geometry has far-reaching consequences. It influenced the later works of Poincaré, Picard, Lefstchetz, Hodge and others. 


\section{A Brief History of Kähler Geometry}

\section{by Shing-Tung Yau*}

\section{Contents}

1 The Work of Riemann . . . . . . . . . . . . 1

2 Calabi Conjecture and Kähler-Einstein Metrics 4

2.1 Kähler-Einstein Metrics on Fano

Manifolds .............. 5

2.2 Balanced Metric and Strominger System 6

2.3 Questions of Kähler-Einstein Metrics in

Algebraic Geometry . . . . . . . . . . 7

2.3.1 Understanding of Kähler-Einstein

Metrics near Singularities . . . . 7

2.3.2 Kähler-Einstein Metrics on Quasiprojective Varieties and Sasakian-

Einstein Metrics . . . . . . . . 8

2.3.3 Compatification of Shimura Varieties ............ 8

2.3.4 Explicit Construction of KählerEinstein Metrics and Uniformization . . . . . . . . . . 10

2.3.5 Relation with Birational Geometry 11

3 Hermitian-Yang-Mills Connections . . . . . . . 12

3.1 Donaldson-Uhlenbeck-Yau Correspondence . . . . . . . . . . . . . . . . 12

3.2 Chern Number Inequalities and Characterization of Flat Bundles . . . . . . . . 13

3.3 Generalization to Non-Kähler and NonCompact Manifolds . . . . . . . . . . . 14

3.4 Analytic Criterions for Various Stability Conditions . . . . . . . . . . . . . . . 14

4 Mirror Symmetry . . . . . . . . . . . . . . . . 15

4.1 Counting of Curves . . . . . . . . . . 15

4.2 Mathematical Approaches to Mirror Symmetry . . . . . . . . . . . . . . . 15

5 Future Directions in Mathematical Physics and Arithmetic Geometry . . . . . . . . 16

References . . . . . . . . . . . . . . . . . 17

* Department of Mathematics, Harvard University, Cambridge, Massachusetts, U.S.A.

E-mail: yau@math.harvard.edu

\section{The Work of Riemann}

Riemann was one of the founders of complex analysis, along with Cauchy. Riemann pioneered several directions in the subject of holomorphic functions:

1. The idea of using differential equations and variational principle. The major work here is the Cauchy-Riemann equation, and the creation of Dirichlet principle to solve the boundary value problem for harmonic functions. (It took several great mathematicians, such as David Hilbert, to complete this work of Riemann.)

2. He gave the proof of the Riemann mapping theorem for simply connected domains. This theory of uniformization theorems has been extremely influential. There are methods based on various approaches, including methods of partial differential equations, hypergeometric functions and algebraic geometry. A natural generalization is to understand the moduli space of Riemann surfaces where Riemann made an important contribution by showing that it is a complex variety with dimension $3 g-3$.

3. The idea of using geometry to understand multivalued holomorphic functions, where he looked at the largest domain that a multivalued holomorphic function can define. He created the concept of Riemann surfaces, where he studied their topology and their moduli space. In fact, he introduced the concept of connectivity of space by cutting Riemann surface into pieces. The concept of Betti number was introduced by him for spaces in arbitrary dimension.

The idea of understanding analytic problems through topology or geometry has far-reaching consequences. It influenced the later works of Poincaré, Picard, Lefstchetz, Hodge and others. 


\section{A Brief History of Kähler Geometry}

\section{by Shing-Tung Yau*}

\section{Contents}

1 The Work of Riemann . . . . . . . . . . . . 1

2 Calabi Conjecture and Kähler-Einstein Metrics 4

2.1 Kähler-Einstein Metrics on Fano

Manifolds .............. 5

2.2 Balanced Metric and Strominger System 6

2.3 Questions of Kähler-Einstein Metrics in

Algebraic Geometry . . . . . . . . . . 7

2.3.1 Understanding of Kähler-Einstein

Metrics near Singularities . . . . 7

2.3.2 Kähler-Einstein Metrics on Quasiprojective Varieties and Sasakian-

Einstein Metrics . . . . . . . . 8

2.3.3 Compatification of Shimura Varieties ............ 8

2.3.4 Explicit Construction of KählerEinstein Metrics and Uniformization . . . . . . . . . . 10

2.3.5 Relation with Birational Geometry 11

3 Hermitian-Yang-Mills Connections . . . . . . . 12

3.1 Donaldson-Uhlenbeck-Yau Correspondence . . . . . . . . . . . . . . . . 12

3.2 Chern Number Inequalities and Characterization of Flat Bundles . . . . . . . . 13

3.3 Generalization to Non-Kähler and NonCompact Manifolds . . . . . . . . . . . 14

3.4 Analytic Criterions for Various Stability Conditions . . . . . . . . . . . . . . . 14

4 Mirror Symmetry . . . . . . . . . . . . . . . . 15

4.1 Counting of Curves . . . . . . . . . . 15

4.2 Mathematical Approaches to Mirror Symmetry . . . . . . . . . . . . . . . 15

5 Future Directions in Mathematical Physics and Arithmetic Geometry . . . . . . . . 16

References . . . . . . . . . . . . . . . . . 17

* Department of Mathematics, Harvard University, Cambridge, Massachusetts, U.S.A.

E-mail: yau@math.harvard.edu

\section{The Work of Riemann}

Riemann was one of the founders of complex analysis, along with Cauchy. Riemann pioneered several directions in the subject of holomorphic functions:

1. The idea of using differential equations and variational principle. The major work here is the Cauchy-Riemann equation, and the creation of Dirichlet principle to solve the boundary value problem for harmonic functions. (It took several great mathematicians, such as David Hilbert, to complete this work of Riemann.)

2. He gave the proof of the Riemann mapping theorem for simply connected domains. This theory of uniformization theorems has been extremely influential. There are methods based on various approaches, including methods of partial differential equations, hypergeometric functions and algebraic geometry. A natural generalization is to understand the moduli space of Riemann surfaces where Riemann made an important contribution by showing that it is a complex variety with dimension $3 g-3$.

3. The idea of using geometry to understand multivalued holomorphic functions, where he looked at the largest domain that a multivalued holomorphic function can define. He created the concept of Riemann surfaces, where he studied their topology and their moduli space. In fact, he introduced the concept of connectivity of space by cutting Riemann surface into pieces. The concept of Betti number was introduced by him for spaces in arbitrary dimension.

The idea of understanding analytic problems through topology or geometry has far-reaching consequences. It influenced the later works of Poincaré, Picard, Lefstchetz, Hodge and others. 


\section{A Brief History of Kähler Geometry}

\section{by Shing-Tung Yau*}

\section{Contents}

1 The Work of Riemann . . . . . . . . . . . . 1

2 Calabi Conjecture and Kähler-Einstein Metrics 4

2.1 Kähler-Einstein Metrics on Fano

Manifolds .............. 5

2.2 Balanced Metric and Strominger System 6

2.3 Questions of Kähler-Einstein Metrics in

Algebraic Geometry . . . . . . . . . . 7

2.3.1 Understanding of Kähler-Einstein

Metrics near Singularities . . . . 7

2.3.2 Kähler-Einstein Metrics on Quasiprojective Varieties and Sasakian-

Einstein Metrics . . . . . . . . 8

2.3.3 Compatification of Shimura Varieties ............ 8

2.3.4 Explicit Construction of KählerEinstein Metrics and Uniformization . . . . . . . . . . 10

2.3.5 Relation with Birational Geometry 11

3 Hermitian-Yang-Mills Connections . . . . . . . 12

3.1 Donaldson-Uhlenbeck-Yau Correspondence . . . . . . . . . . . . . . . . 12

3.2 Chern Number Inequalities and Characterization of Flat Bundles . . . . . . . . 13

3.3 Generalization to Non-Kähler and NonCompact Manifolds . . . . . . . . . . . 14

3.4 Analytic Criterions for Various Stability Conditions . . . . . . . . . . . . . . . 14

4 Mirror Symmetry . . . . . . . . . . . . . . . . 15

4.1 Counting of Curves . . . . . . . . . . 15

4.2 Mathematical Approaches to Mirror Symmetry . . . . . . . . . . . . . . . 15

5 Future Directions in Mathematical Physics and Arithmetic Geometry . . . . . . . . 16

References . . . . . . . . . . . . . . . . . 17

* Department of Mathematics, Harvard University, Cambridge, Massachusetts, U.S.A.

E-mail: yau@math.harvard.edu

\section{The Work of Riemann}

Riemann was one of the founders of complex analysis, along with Cauchy. Riemann pioneered several directions in the subject of holomorphic functions:

1. The idea of using differential equations and variational principle. The major work here is the Cauchy-Riemann equation, and the creation of Dirichlet principle to solve the boundary value problem for harmonic functions. (It took several great mathematicians, such as David Hilbert, to complete this work of Riemann.)

2. He gave the proof of the Riemann mapping theorem for simply connected domains. This theory of uniformization theorems has been extremely influential. There are methods based on various approaches, including methods of partial differential equations, hypergeometric functions and algebraic geometry. A natural generalization is to understand the moduli space of Riemann surfaces where Riemann made an important contribution by showing that it is a complex variety with dimension $3 g-3$.

3. The idea of using geometry to understand multivalued holomorphic functions, where he looked at the largest domain that a multivalued holomorphic function can define. He created the concept of Riemann surfaces, where he studied their topology and their moduli space. In fact, he introduced the concept of connectivity of space by cutting Riemann surface into pieces. The concept of Betti number was introduced by him for spaces in arbitrary dimension.

The idea of understanding analytic problems through topology or geometry has far-reaching consequences. It influenced the later works of Poincaré, Picard, Lefstchetz, Hodge and others. 


\section{A Brief History of Kähler Geometry}

\section{by Shing-Tung Yau*}

\section{Contents}

1 The Work of Riemann . . . . . . . . . . . . 1

2 Calabi Conjecture and Kähler-Einstein Metrics 4

2.1 Kähler-Einstein Metrics on Fano

Manifolds .............. 5

2.2 Balanced Metric and Strominger System 6

2.3 Questions of Kähler-Einstein Metrics in

Algebraic Geometry . . . . . . . . . . 7

2.3.1 Understanding of Kähler-Einstein

Metrics near Singularities . . . . 7

2.3.2 Kähler-Einstein Metrics on Quasiprojective Varieties and Sasakian-

Einstein Metrics . . . . . . . . 8

2.3.3 Compatification of Shimura Varieties ............ 8

2.3.4 Explicit Construction of KählerEinstein Metrics and Uniformization . . . . . . . . . . 10

2.3.5 Relation with Birational Geometry 11

3 Hermitian-Yang-Mills Connections . . . . . . . 12

3.1 Donaldson-Uhlenbeck-Yau Correspondence . . . . . . . . . . . . . . . . 12

3.2 Chern Number Inequalities and Characterization of Flat Bundles . . . . . . . . 13

3.3 Generalization to Non-Kähler and NonCompact Manifolds . . . . . . . . . . . 14

3.4 Analytic Criterions for Various Stability Conditions . . . . . . . . . . . . . . . 14

4 Mirror Symmetry . . . . . . . . . . . . . . . . 15

4.1 Counting of Curves . . . . . . . . . . 15

4.2 Mathematical Approaches to Mirror Symmetry . . . . . . . . . . . . . . . 15

5 Future Directions in Mathematical Physics and Arithmetic Geometry . . . . . . . . 16

References . . . . . . . . . . . . . . . . . 17

* Department of Mathematics, Harvard University, Cambridge, Massachusetts, U.S.A.

E-mail: yau@math.harvard.edu

\section{The Work of Riemann}

Riemann was one of the founders of complex analysis, along with Cauchy. Riemann pioneered several directions in the subject of holomorphic functions:

1. The idea of using differential equations and variational principle. The major work here is the Cauchy-Riemann equation, and the creation of Dirichlet principle to solve the boundary value problem for harmonic functions. (It took several great mathematicians, such as David Hilbert, to complete this work of Riemann.)

2. He gave the proof of the Riemann mapping theorem for simply connected domains. This theory of uniformization theorems has been extremely influential. There are methods based on various approaches, including methods of partial differential equations, hypergeometric functions and algebraic geometry. A natural generalization is to understand the moduli space of Riemann surfaces where Riemann made an important contribution by showing that it is a complex variety with dimension $3 g-3$.

3. The idea of using geometry to understand multivalued holomorphic functions, where he looked at the largest domain that a multivalued holomorphic function can define. He created the concept of Riemann surfaces, where he studied their topology and their moduli space. In fact, he introduced the concept of connectivity of space by cutting Riemann surface into pieces. The concept of Betti number was introduced by him for spaces in arbitrary dimension.

The idea of understanding analytic problems through topology or geometry has far-reaching consequences. It influenced the later works of Poincaré, Picard, Lefstchetz, Hodge and others. 


\section{A Brief History of Kähler Geometry}

\section{by Shing-Tung Yau*}

\section{Contents}

1 The Work of Riemann . . . . . . . . . . . . 1

2 Calabi Conjecture and Kähler-Einstein Metrics 4

2.1 Kähler-Einstein Metrics on Fano

Manifolds .............. 5

2.2 Balanced Metric and Strominger System 6

2.3 Questions of Kähler-Einstein Metrics in

Algebraic Geometry . . . . . . . . . . 7

2.3.1 Understanding of Kähler-Einstein

Metrics near Singularities . . . . 7

2.3.2 Kähler-Einstein Metrics on Quasiprojective Varieties and Sasakian-

Einstein Metrics . . . . . . . . 8

2.3.3 Compatification of Shimura Varieties ............ 8

2.3.4 Explicit Construction of KählerEinstein Metrics and Uniformization . . . . . . . . . . 10

2.3.5 Relation with Birational Geometry 11

3 Hermitian-Yang-Mills Connections . . . . . . . 12

3.1 Donaldson-Uhlenbeck-Yau Correspondence . . . . . . . . . . . . . . . . 12

3.2 Chern Number Inequalities and Characterization of Flat Bundles . . . . . . . . 13

3.3 Generalization to Non-Kähler and NonCompact Manifolds . . . . . . . . . . . 14

3.4 Analytic Criterions for Various Stability Conditions . . . . . . . . . . . . . . . 14

4 Mirror Symmetry . . . . . . . . . . . . . . . . 15

4.1 Counting of Curves . . . . . . . . . . 15

4.2 Mathematical Approaches to Mirror Symmetry . . . . . . . . . . . . . . . 15

5 Future Directions in Mathematical Physics and Arithmetic Geometry . . . . . . . . 16

References . . . . . . . . . . . . . . . . . 17

* Department of Mathematics, Harvard University, Cambridge, Massachusetts, U.S.A.

E-mail: yau@math.harvard.edu

\section{The Work of Riemann}

Riemann was one of the founders of complex analysis, along with Cauchy. Riemann pioneered several directions in the subject of holomorphic functions:

1. The idea of using differential equations and variational principle. The major work here is the Cauchy-Riemann equation, and the creation of Dirichlet principle to solve the boundary value problem for harmonic functions. (It took several great mathematicians, such as David Hilbert, to complete this work of Riemann.)

2. He gave the proof of the Riemann mapping theorem for simply connected domains. This theory of uniformization theorems has been extremely influential. There are methods based on various approaches, including methods of partial differential equations, hypergeometric functions and algebraic geometry. A natural generalization is to understand the moduli space of Riemann surfaces where Riemann made an important contribution by showing that it is a complex variety with dimension $3 g-3$.

3. The idea of using geometry to understand multivalued holomorphic functions, where he looked at the largest domain that a multivalued holomorphic function can define. He created the concept of Riemann surfaces, where he studied their topology and their moduli space. In fact, he introduced the concept of connectivity of space by cutting Riemann surface into pieces. The concept of Betti number was introduced by him for spaces in arbitrary dimension.

The idea of understanding analytic problems through topology or geometry has far-reaching consequences. It influenced the later works of Poincaré, Picard, Lefstchetz, Hodge and others. 


\section{A Brief History of Kähler Geometry}

\section{by Shing-Tung Yau*}

\section{Contents}

1 The Work of Riemann . . . . . . . . . . . . 1

2 Calabi Conjecture and Kähler-Einstein Metrics 4

2.1 Kähler-Einstein Metrics on Fano

Manifolds .............. 5

2.2 Balanced Metric and Strominger System 6

2.3 Questions of Kähler-Einstein Metrics in

Algebraic Geometry . . . . . . . . . . 7

2.3.1 Understanding of Kähler-Einstein

Metrics near Singularities . . . . 7

2.3.2 Kähler-Einstein Metrics on Quasiprojective Varieties and Sasakian-

Einstein Metrics . . . . . . . . 8

2.3.3 Compatification of Shimura Varieties ............ 8

2.3.4 Explicit Construction of KählerEinstein Metrics and Uniformization . . . . . . . . . . 10

2.3.5 Relation with Birational Geometry 11

3 Hermitian-Yang-Mills Connections . . . . . . . 12

3.1 Donaldson-Uhlenbeck-Yau Correspondence . . . . . . . . . . . . . . . . 12

3.2 Chern Number Inequalities and Characterization of Flat Bundles . . . . . . . . 13

3.3 Generalization to Non-Kähler and NonCompact Manifolds . . . . . . . . . . . 14

3.4 Analytic Criterions for Various Stability Conditions . . . . . . . . . . . . . . . 14

4 Mirror Symmetry . . . . . . . . . . . . . . . . 15

4.1 Counting of Curves . . . . . . . . . . 15

4.2 Mathematical Approaches to Mirror Symmetry . . . . . . . . . . . . . . . 15

5 Future Directions in Mathematical Physics and Arithmetic Geometry . . . . . . . . 16

References . . . . . . . . . . . . . . . . . 17

* Department of Mathematics, Harvard University, Cambridge, Massachusetts, U.S.A.

E-mail: yau@math.harvard.edu

\section{The Work of Riemann}

Riemann was one of the founders of complex analysis, along with Cauchy. Riemann pioneered several directions in the subject of holomorphic functions:

1. The idea of using differential equations and variational principle. The major work here is the Cauchy-Riemann equation, and the creation of Dirichlet principle to solve the boundary value problem for harmonic functions. (It took several great mathematicians, such as David Hilbert, to complete this work of Riemann.)

2. He gave the proof of the Riemann mapping theorem for simply connected domains. This theory of uniformization theorems has been extremely influential. There are methods based on various approaches, including methods of partial differential equations, hypergeometric functions and algebraic geometry. A natural generalization is to understand the moduli space of Riemann surfaces where Riemann made an important contribution by showing that it is a complex variety with dimension $3 g-3$.

3. The idea of using geometry to understand multivalued holomorphic functions, where he looked at the largest domain that a multivalued holomorphic function can define. He created the concept of Riemann surfaces, where he studied their topology and their moduli space. In fact, he introduced the concept of connectivity of space by cutting Riemann surface into pieces. The concept of Betti number was introduced by him for spaces in arbitrary dimension.

The idea of understanding analytic problems through topology or geometry has far-reaching consequences. It influenced the later works of Poincaré, Picard, Lefstchetz, Hodge and others. 


\section{A Brief History of Kähler Geometry}

\section{by Shing-Tung Yau*}

\section{Contents}

1 The Work of Riemann . . . . . . . . . . . . 1

2 Calabi Conjecture and Kähler-Einstein Metrics 4

2.1 Kähler-Einstein Metrics on Fano

Manifolds .............. 5

2.2 Balanced Metric and Strominger System 6

2.3 Questions of Kähler-Einstein Metrics in

Algebraic Geometry . . . . . . . . . . 7

2.3.1 Understanding of Kähler-Einstein

Metrics near Singularities . . . . 7

2.3.2 Kähler-Einstein Metrics on Quasiprojective Varieties and Sasakian-

Einstein Metrics . . . . . . . . 8

2.3.3 Compatification of Shimura Varieties ............ 8

2.3.4 Explicit Construction of KählerEinstein Metrics and Uniformization . . . . . . . . . . 10

2.3.5 Relation with Birational Geometry 11

3 Hermitian-Yang-Mills Connections . . . . . . . 12

3.1 Donaldson-Uhlenbeck-Yau Correspondence . . . . . . . . . . . . . . . . 12

3.2 Chern Number Inequalities and Characterization of Flat Bundles . . . . . . . . 13

3.3 Generalization to Non-Kähler and NonCompact Manifolds . . . . . . . . . . . 14

3.4 Analytic Criterions for Various Stability Conditions . . . . . . . . . . . . . . . 14

4 Mirror Symmetry . . . . . . . . . . . . . . . . 15

4.1 Counting of Curves . . . . . . . . . . 15

4.2 Mathematical Approaches to Mirror Symmetry . . . . . . . . . . . . . . . 15

5 Future Directions in Mathematical Physics and Arithmetic Geometry . . . . . . . . 16

References . . . . . . . . . . . . . . . . . 17

* Department of Mathematics, Harvard University, Cambridge, Massachusetts, U.S.A.

E-mail: yau@math.harvard.edu

\section{The Work of Riemann}

Riemann was one of the founders of complex analysis, along with Cauchy. Riemann pioneered several directions in the subject of holomorphic functions:

1. The idea of using differential equations and variational principle. The major work here is the Cauchy-Riemann equation, and the creation of Dirichlet principle to solve the boundary value problem for harmonic functions. (It took several great mathematicians, such as David Hilbert, to complete this work of Riemann.)

2. He gave the proof of the Riemann mapping theorem for simply connected domains. This theory of uniformization theorems has been extremely influential. There are methods based on various approaches, including methods of partial differential equations, hypergeometric functions and algebraic geometry. A natural generalization is to understand the moduli space of Riemann surfaces where Riemann made an important contribution by showing that it is a complex variety with dimension $3 g-3$.

3. The idea of using geometry to understand multivalued holomorphic functions, where he looked at the largest domain that a multivalued holomorphic function can define. He created the concept of Riemann surfaces, where he studied their topology and their moduli space. In fact, he introduced the concept of connectivity of space by cutting Riemann surface into pieces. The concept of Betti number was introduced by him for spaces in arbitrary dimension.

The idea of understanding analytic problems through topology or geometry has far-reaching consequences. It influenced the later works of Poincaré, Picard, Lefstchetz, Hodge and others. 


\section{A Brief History of Kähler Geometry}

\section{by Shing-Tung Yau*}

\section{Contents}

1 The Work of Riemann . . . . . . . . . . . . 1

2 Calabi Conjecture and Kähler-Einstein Metrics 4

2.1 Kähler-Einstein Metrics on Fano

Manifolds .............. 5

2.2 Balanced Metric and Strominger System 6

2.3 Questions of Kähler-Einstein Metrics in

Algebraic Geometry . . . . . . . . . . 7

2.3.1 Understanding of Kähler-Einstein

Metrics near Singularities . . . . 7

2.3.2 Kähler-Einstein Metrics on Quasiprojective Varieties and Sasakian-

Einstein Metrics . . . . . . . . 8

2.3.3 Compatification of Shimura Varieties ............ 8

2.3.4 Explicit Construction of KählerEinstein Metrics and Uniformization . . . . . . . . . . 10

2.3.5 Relation with Birational Geometry 11

3 Hermitian-Yang-Mills Connections . . . . . . . 12

3.1 Donaldson-Uhlenbeck-Yau Correspondence . . . . . . . . . . . . . . . . 12

3.2 Chern Number Inequalities and Characterization of Flat Bundles . . . . . . . . 13

3.3 Generalization to Non-Kähler and NonCompact Manifolds . . . . . . . . . . . 14

3.4 Analytic Criterions for Various Stability Conditions . . . . . . . . . . . . . . . 14

4 Mirror Symmetry . . . . . . . . . . . . . . . . 15

4.1 Counting of Curves . . . . . . . . . . 15

4.2 Mathematical Approaches to Mirror Symmetry . . . . . . . . . . . . . . . 15

5 Future Directions in Mathematical Physics and Arithmetic Geometry . . . . . . . . 16

References . . . . . . . . . . . . . . . . . 17

* Department of Mathematics, Harvard University, Cambridge, Massachusetts, U.S.A.

E-mail: yau@math.harvard.edu

\section{The Work of Riemann}

Riemann was one of the founders of complex analysis, along with Cauchy. Riemann pioneered several directions in the subject of holomorphic functions:

1. The idea of using differential equations and variational principle. The major work here is the Cauchy-Riemann equation, and the creation of Dirichlet principle to solve the boundary value problem for harmonic functions. (It took several great mathematicians, such as David Hilbert, to complete this work of Riemann.)

2. He gave the proof of the Riemann mapping theorem for simply connected domains. This theory of uniformization theorems has been extremely influential. There are methods based on various approaches, including methods of partial differential equations, hypergeometric functions and algebraic geometry. A natural generalization is to understand the moduli space of Riemann surfaces where Riemann made an important contribution by showing that it is a complex variety with dimension $3 g-3$.

3. The idea of using geometry to understand multivalued holomorphic functions, where he looked at the largest domain that a multivalued holomorphic function can define. He created the concept of Riemann surfaces, where he studied their topology and their moduli space. In fact, he introduced the concept of connectivity of space by cutting Riemann surface into pieces. The concept of Betti number was introduced by him for spaces in arbitrary dimension.

The idea of understanding analytic problems through topology or geometry has far-reaching consequences. It influenced the later works of Poincaré, Picard, Lefstchetz, Hodge and others. 


\section{A Brief History of Kähler Geometry}

\section{by Shing-Tung Yau*}

\section{Contents}

1 The Work of Riemann . . . . . . . . . . . . 1

2 Calabi Conjecture and Kähler-Einstein Metrics 4

2.1 Kähler-Einstein Metrics on Fano

Manifolds .............. 5

2.2 Balanced Metric and Strominger System 6

2.3 Questions of Kähler-Einstein Metrics in

Algebraic Geometry . . . . . . . . . . 7

2.3.1 Understanding of Kähler-Einstein

Metrics near Singularities . . . . 7

2.3.2 Kähler-Einstein Metrics on Quasiprojective Varieties and Sasakian-

Einstein Metrics . . . . . . . . 8

2.3.3 Compatification of Shimura Varieties ............ 8

2.3.4 Explicit Construction of KählerEinstein Metrics and Uniformization . . . . . . . . . . 10

2.3.5 Relation with Birational Geometry 11

3 Hermitian-Yang-Mills Connections . . . . . . . 12

3.1 Donaldson-Uhlenbeck-Yau Correspondence . . . . . . . . . . . . . . . . 12

3.2 Chern Number Inequalities and Characterization of Flat Bundles . . . . . . . . 13

3.3 Generalization to Non-Kähler and NonCompact Manifolds . . . . . . . . . . . 14

3.4 Analytic Criterions for Various Stability Conditions . . . . . . . . . . . . . . . 14

4 Mirror Symmetry . . . . . . . . . . . . . . . . 15

4.1 Counting of Curves . . . . . . . . . . 15

4.2 Mathematical Approaches to Mirror Symmetry . . . . . . . . . . . . . . . 15

5 Future Directions in Mathematical Physics and Arithmetic Geometry . . . . . . . . 16

References . . . . . . . . . . . . . . . . . 17

* Department of Mathematics, Harvard University, Cambridge, Massachusetts, U.S.A.

E-mail: yau@math.harvard.edu

\section{The Work of Riemann}

Riemann was one of the founders of complex analysis, along with Cauchy. Riemann pioneered several directions in the subject of holomorphic functions:

1. The idea of using differential equations and variational principle. The major work here is the Cauchy-Riemann equation, and the creation of Dirichlet principle to solve the boundary value problem for harmonic functions. (It took several great mathematicians, such as David Hilbert, to complete this work of Riemann.)

2. He gave the proof of the Riemann mapping theorem for simply connected domains. This theory of uniformization theorems has been extremely influential. There are methods based on various approaches, including methods of partial differential equations, hypergeometric functions and algebraic geometry. A natural generalization is to understand the moduli space of Riemann surfaces where Riemann made an important contribution by showing that it is a complex variety with dimension $3 g-3$.

3. The idea of using geometry to understand multivalued holomorphic functions, where he looked at the largest domain that a multivalued holomorphic function can define. He created the concept of Riemann surfaces, where he studied their topology and their moduli space. In fact, he introduced the concept of connectivity of space by cutting Riemann surface into pieces. The concept of Betti number was introduced by him for spaces in arbitrary dimension.

The idea of understanding analytic problems through topology or geometry has far-reaching consequences. It influenced the later works of Poincaré, Picard, Lefstchetz, Hodge and others. 